\title{
Dendritic cell subpopulations in nasopharyngeal cancer
}

\author{
JOHAN S. NILSSON ${ }^{1,2^{*}}$, MILAD ABOLHALAJ $^{3 *}$, KRISTINA LUNDBERG $^{3}$, \\ MALIN LINDSTEDT ${ }^{3}$ and LENNART GREIFF ${ }^{1,2}$ \\ ${ }^{1}$ Department of Otorhinolaryngology, Head and Neck Surgery, Skåne University Hospital, Lund SE-221 85; \\ Departments of ${ }^{2}$ Clinical Sciences and ${ }^{3}$ Immunotechnology, Lund University, Lund SE-221 00, Sweden
}

Received April 30, 2018; Accepted September 17, 2018

DOI: $10.3892 / \mathrm{ol} .2018 .9835$

\begin{abstract}
Nasopharyngeal cancer (NPC) is associated with Epstein-Barr virus (EBV) and EBV antigen may be utilized for therapeutic purposes, including targeting of dendritic cells (DCs). Although DCs may be present in NPC, the information is limited and not up-to-date with current knowledge on DC subsets. In the present study, biopsies from untreated NPC were obtained and subjected to multicolor flow-cytometry focusing on DC subtype markers: CD123 for plasmacytoid DCs (pDCs); and CD1c and CD141 for myeloid DCs (mDCs). Furthermore, subset-specific expression of the $\mathrm{C}$-lectin receptor (CLR) CD207 (also termed langerin) was assessed. pDCs and mDCs were detected in the NPC lesions, contributing to a frequency mean average of $0.78 \%$ of $\mathrm{CD} 45^{+}$leukocytes in situ. Different subpopulations, previously not described in NPC, were observed, including: $\mathrm{CD}_{123}{ }^{+} \mathrm{pDCs}$; $\mathrm{CD}^{+} \mathrm{c}^{+} \mathrm{mDCs}$; $\mathrm{CD} 141^{+} \mathrm{mDCs}$; and $\mathrm{CD} 1 \mathrm{c}^{-} \mathrm{CD} 141^{-} \mathrm{mDCs}$. A high frequency of CD1 $\mathrm{c}^{+}$mDCs expressing CD207 was observed, compared with other subsets. In conclusion, different DC subsets are present in NPC lesions. The CLR CD207, a selective endocytic marker on $\mathrm{CD} \mathrm{c}^{+} \mathrm{mDCs}$, may be targeted for therapeutic purposes to facilitate cross-presentation of antigens and aid cell-mediated antitumor effects.
\end{abstract}

\section{Introduction}

Nasopharyngeal cancer (NPC) is a relatively rare form of squamous cell carcinoma with vast differences in incidence globally (1). Prior infection with Epstein-Barr virus (EBV) is considered an important etiological factor in regions with endemic NPC, along with genetic susceptibility, and dietary and social factors (1). According to a large study conducted

Correspondence to: Professor Lennart Greiff, Department of Otorhinolaryngology, Head and Neck Surgery, Skåne University Hospital, 21 Lasarettsgatan, Lund SE-221 85, Sweden

E-mail: lennart.greiff@skane.se

*Contributed equally

Key words: antigen presentation, C-lectin receptor, langerin, pattern recognition receptor in Hong Kong (a region with endemic NPC) between 1996 and 2000 , the 5-year disease-specific survival rates range from $65 \%$ to $>92 \%$ depending on the cancer stage (2). The side effects of current primary treatments (radiotherapy with or without chemotherapy) are marked (3). There is therefore a requirement for novel treatment approaches.

The association between NPC and EBV provides an opening for antigen-specific treatment for NPC. Adoptive immunotherapy comprising of allogeneic or autologous cytotoxic T lymphocyte (CTL) therapy, bypassing the antigen-presenting procedure, has been investigated (4-7). For instance, Smith et al (7) demonstrated antitumor effects in patients with metastatic NPC treated with autologous antigen-specific T-cell lines generated in vitro using an adenoviral vector-based vaccine (AdE1-LMPpoly) encoding for multiple EBV antigens. However, active immunotherapy, to the best of our knowledge, has not been utilized in the treatment of NPC.

Identification of a tumor-specific EBV antigen in NPC suggests the possibility that the condition is amendable to specific dendritic cell (DC)-targeting immunotherapy. DCs are professional antigen-presenters and key regulators of T-cell polarization (8). Blood DCs form a heterogeneous population, including $\mathrm{CD}_{12} 3^{+}$plasmacytoid DCs (pDCs) as well as $\mathrm{CD}_{1}{ }^{+}$and $\mathrm{CD} 141^{+}$myeloid DCs (mDCs), and each subset features different pattern recognition receptor (PRR) profiles, including Toll-like receptors (TLRs) and C-lectin receptors (CLRs) (9). Adjuvant actions on a number of these receptors, including the CLR CD207 (also termed langerin), may promote cross-presentation of antigens, which is a necessary step to achieve beneficial cell-mediated cytotoxic effects (10-12). Detailed information on intralesional DC subsets and receptor repertoires is necessary in order to design effective immunotherapy for NPC; however, currently there is limited information available.

The presence of human leukocyte antigen (HLA)-DR ${ }^{+}$and HTA-I ${ }^{+}$cells, which are morphologically and phenotypically similar to antigen-presenting Langerhans cells, has previously been reported in NPC (13). Similarly, cells with features of the Langerhans cell type, morphologically or immunohistochemically using S-100 or HLA-DR/HTA-I (CD1a antibodies), have been demonstrated in NPC (14-18). Furthermore, Braz-Silva et al (14) demonstrated a subset of CD207 $7^{+} \mathrm{DCs}$ infiltrating EBV-infected areas in NPC. However, a detailed description of DC subsets, according to current knowledge in 
the field, is limited for NPC. In the present study, using fresh tumor-samples obtained from patients with untreated NPC, the presence and characteristics of DCs was examined using multicolor flow-cytometry. It was demonstrated that different subpopulations of DCs are present in NPC lesions and that $\mathrm{CD}^{+} \mathrm{c}^{+}$mDCs may have an increased expression of CD207, compared with other subsets.

\section{Materials and methods}

Study design. A total of 5 patients, 3 male and 2 female patients, (range, 39-67 years of age) with untreated NPC were recruited between November 2014 and August 2016 at Skåne University Hospital (Lund, Sweden), a tertiary referral center with a catchment population of approximately 1.9 million. The inclusion criteria of the present study was age $>18$ years and no prior cancer treatment. Patient characteristics are presented in Table I, including histopathology classification according to World Health Organization (WHO) (19) and cancer stage according to Tumor-Node-Metastasis (TNM) classification of malignant tumors (20). The Ethics Committee at Lund University (Lund, Sweden) approved the study protocol and written informed consent was obtained from all patients prior to inclusion.

Sampling. Topical anesthesia and mucosal decongestion was achieved by nasal/nasopharyngeal administration of a mixture of tetracain $(20 \mathrm{mg} / \mathrm{ml})$ and adrenalin $(0.1 \mathrm{mg} / \mathrm{ml})$ using a spray-device. Tissue samples were obtained using a punch forceps under endoscopic guidance. Half of the biopsy was sent for routine pathology work, including EBV-encoded small RNAs-1 (EBER1) in situ hybridization. The other half was stored in tissue storage solution at $4^{\circ} \mathrm{C}$ (Miltenyi Biotec $\mathrm{GmbH}$, Bergisch Gladbach, Germany) and transferred to the laboratory.

Cell preparation. Fresh biopsies were cut into $\sim 2 \mathrm{~mm}$ pieces and placed in RPMI-1640 medium (Thermo Fisher Scientific, Inc., Waltham, MA, USA) supplemented with gentamycin (0.1 g/ml; Sigma-Aldrich; Merck KGaA, Darmstadt, Germany). Enzymatic digestion was performed by incubating the tissue suspension at $37^{\circ} \mathrm{C}$ for $20 \mathrm{~min}$ with Collagenase IV $(2 \mathrm{mg} / \mathrm{ml})$ and DNase I (200 Kunitz/ml) (both from Sigma-Aldrich; Merck KGaA). A single cell suspension was prepared by filtering the cell suspension through $70 \mu \mathrm{m}$ cell strainers (BD Biosciences, San Jose, CA, USA). The number of viable cells were calculated using 4\% Trypan blue solution (Thermo Fisher Scientific, Inc.) and non-stained cells were counted instantly using Fluovert FS inverted fluorescence microscope (magnification, x20; Leitz). Cell viability was evaluated using Trypan blue-exclusion.

Multicolor flow-cytometry. Single cell suspension was blocked using mouse $\mathrm{IgG}$ (Table II) and incubated for $5 \mathrm{~min}$ at room temperature, followed by incubation at $4^{\circ} \mathrm{C}$ for $20 \mathrm{~min}$ in Brilliant Stain Buffer (BD Biosciences) in the presence of the antibody panel indicated in Table II. Stained cells were run on a BD FACSAria II (BD Biosciences) and subset frequencies as well as CD207 expression was further analyzed with FCS Express v4.0 (De Novo Software, Glendale, CA, USA).
Viable cells were gated in a forward scatter (FSC)-side scatter (SSC) plot and doublet exclusion was then performed using FSC-area vs. FSC-height. Leukocytes were subsequently identified as $\mathrm{CD}^{+} 5^{+}$cells, out of which HLA-DR ${ }^{+}$lineage(CD3, CD14, CD56 and CD19) cells were gated. mDCs and pDCs were identified based on the expression of CD11c and CD123, respectively, and these subsets collectively were the total number of DCs. mDCs were further subdivided into $\mathrm{CD}_{141}{ }^{+}, \mathrm{CD}_{1 \mathrm{c}^{+}}$and $\mathrm{CD} 1 \mathrm{c}^{-} \mathrm{CD} 141^{-}$cells. The applied gating strategy is indicated in Fig. 1. Cell surface expression of CD207 was assessed using a similar gating strategy (Fig. 1). A fluorescence minus one (FMO) control was used for the PE channel (an identical additional sample prepared without CD207-staining antibodies) when sufficient numbers of cells were available to perform two staining preparations from the same sample (the DC populations are present in very low frequencies and $\geq 1 \times 10^{6}$ live $\mathrm{CD} 45^{+}$cells are required in order to distinguish the populations and to gate $\mathrm{CD} 207^{+}$cells). The gate for $\mathrm{CD} 207^{+}$cells was set based on the FMO sample if available; otherwise the gating was compared with samples with an FMO to ensure appropriate gate settings.

Statistics. Descriptive data was presented as mean \pm standard deviation where appropriate. A $\chi^{2}$ test was performed to analyze the difference in DC subset frequencies. The statistical analysis was performed in GraphPad prism 6.0 software (GraphPad Software, La Jolla, CA) and VassarStats (vassarstats.net). $\mathrm{P}<0.05$ was considered to indicate a statistically significant difference.

\section{Results}

DCs, including $\mathrm{CD} 11 \mathrm{c}^{+} \mathrm{mDC}$ and $\mathrm{CD} 123^{+} \mathrm{pDCs}$, were detected in all NPC lesions $(n=5)$, contributing to a frequency mean average of $0.78 \pm 0.50 \%$ out of the CD $45^{+}$leukocytes in situ (Fig. 2A). A significant difference was observed between the $\mathrm{CD}_{123^{+}} \mathrm{pDCs}$ and $\mathrm{CD} 11 \mathrm{c}^{+}$mDCs $(\mathrm{P}<0.01$; Fig. 2B), indicating a different presence of aforementioned DCs in the lesions.

Different DC subpopulations, including CD123+ $\mathrm{pDCs}$,

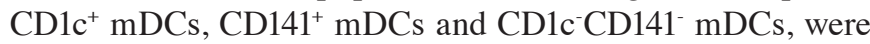
observed, constituting 44.27 $\pm 9.78,21.17 \pm 10.21,6.42 \pm 1.84$ and $24.03 \pm 9.79 \%$ of DCs, respectively (Fig. 2C), and the differences in frequency in these subpopulations were significant $(\mathrm{P}<0.001)$. CD207 expression in the different subsets was increased in $\mathrm{CD}^{+} \mathrm{c}^{+} \mathrm{DCs}(\mathrm{n}=2)$, suggesting a selective expression of CD207 in this subset (Fig. 2D).

\section{Discussion}

In the present study, an intralesional presence of DCs in untreated NPC and their subpopulation frequencies was demonstrated. This includes $\mathrm{CD} 1 \mathrm{c}^{+}$and $\mathrm{CD} 141^{+} \mathrm{mDCs}$ that are of interest in the context of cross-presentation of antigens (21). Furthermore, a notable expression of the CLR CD207 in $\mathrm{CD}^{+} \mathrm{c}^{+} \mathrm{mDC}$ is suggested, which is of interest for the identical reason. The results are of relevance to future study designs and to therapeutic attempts to instruct DCs to facilitate antigen-specific immunological responses against NPC.

The present data demonstrates that DCs are present in NPC, which verifies previous morphological observations (13-18). 
Table I. Patient characteristics.

\begin{tabular}{lccccc}
\hline Sex & Age, years & EBER-1 & Histopathology $^{\mathrm{a}}$ & TNM $^{\mathrm{b}}$ & TNM stage $^{\mathrm{b}}$ \\
\hline Female & 67 & Negative & 1 & T2N3bM1 & IV-C \\
Female & 40 & Positive & $2 \mathrm{~b}$ & T4N1M0 & IV-A \\
Male & 39 & Positive & $2 \mathrm{~b}$ & T1N1M0 & II \\
Male & 65 & Positive & $2 \mathrm{~b}$ & T1N1M0 & II \\
Male & 46 & Positive & $2 \mathrm{~b}$ & T3N2M0 & III
\end{tabular}

${ }^{a}$ World Health Organization histopathology classification of nasopharyngeal cancer 2005 (19): 1) Keratinizing squamous cell carcinoma;

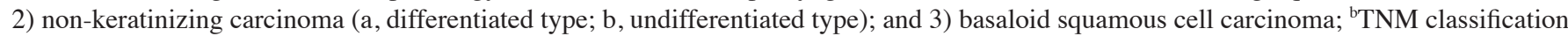
of malignant tumors (20). EBER-1, EBV-encoded small RNAs 1; TNM, tumor-node-metastasis.

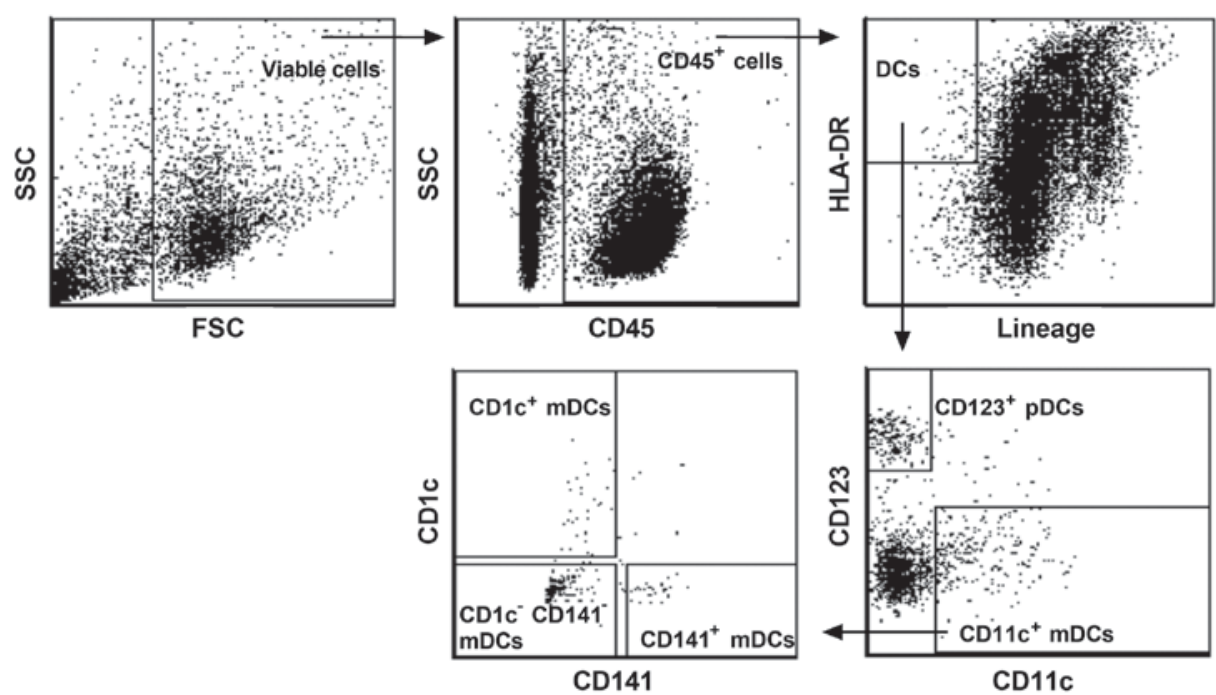

Figure 1. Flow-cytometry gating-strategy exemplified with results for one Epstein-Barr Virus-positive nasopharyngeal cancer tissue sample. Clear separations were observed for DC populations. However, several were scarce in number, notably CD1c ${ }^{+}$and CD $141^{+}$myeloid DCs. DC, dendritic cell; FSC, forward scatter; SSC, side scatter; HLA-DR, human leukocyte antigen-DR.
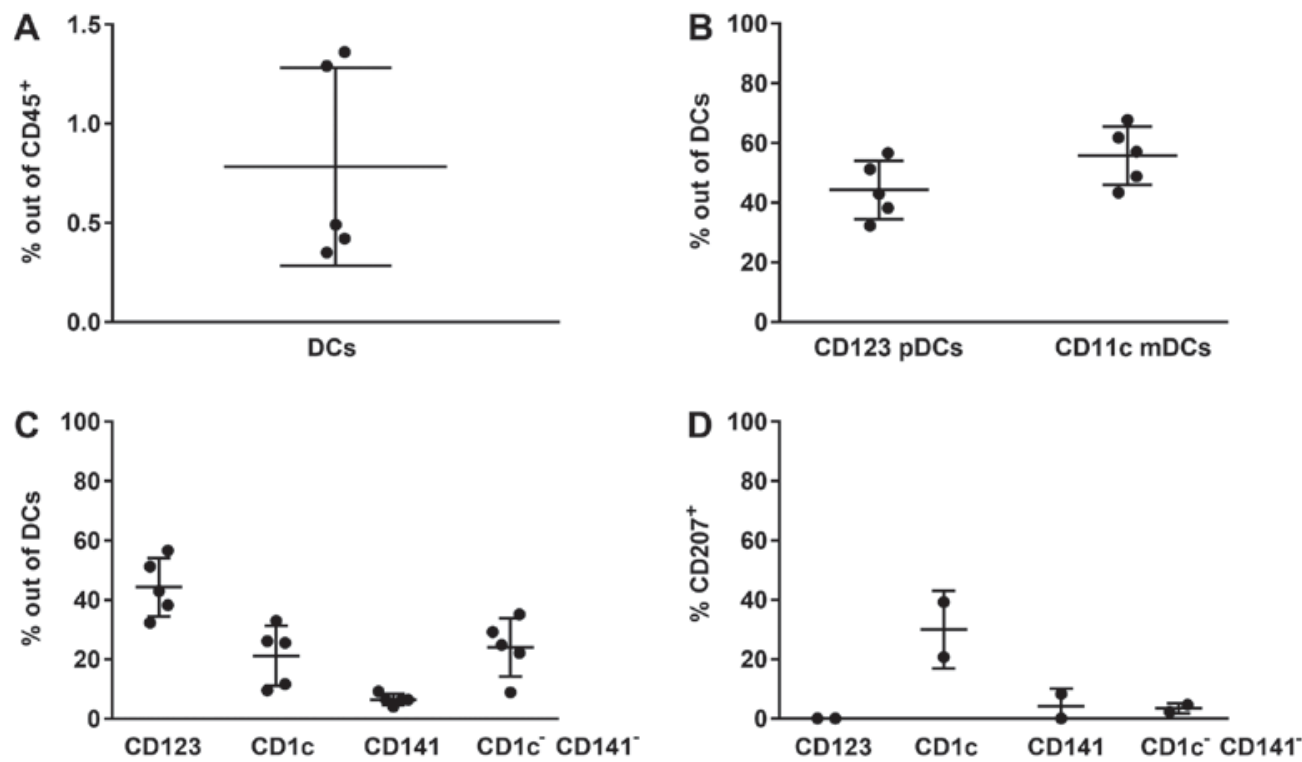

Figure 2. (A) Percentage of DCs out of the total number of CD45 leukocytes. The NPC lesions all featured DCs. (B) Percentage of CD123+ and CD11 $\mathrm{c}^{+} \mathrm{DCs}$ out of the total numbers of DCs. In the NPC lesions, pDCs as well as mDCs were present. (C) Percentage of DC subpopulations out of the total numbers of DCs. (D) Percentage of CD207+ DCs per subpopulation. CD207 expression was exhibited in CD1c $\mathrm{c}^{+} \mathrm{mDCs}$, whereas it was low in CD141 ${ }^{+}$and $\mathrm{CD} 141^{-} \mathrm{CD} 1 \mathrm{c}$ mDCs, but absent in CD123 ${ }^{+}$pDCs. Data are presented in scatter plots as mean \pm standard deviation. The tumor samples in these cases were Epstein-Barr Virus-positive. DCs, dendritic cells; NPC, nasopharyngeal cancer; pDCs, plasmacytoid DCs; mDCs, myeloid DCs. 


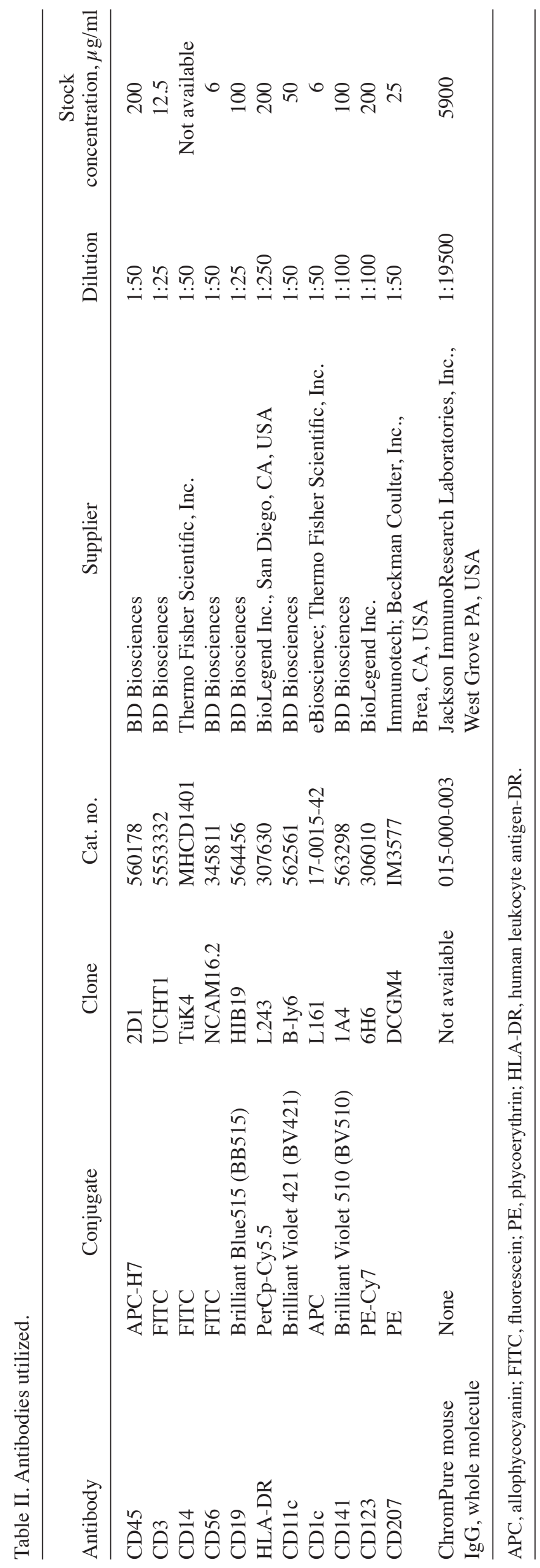

Furthermore, it was indicated for the first time that specific subpopulations, previously characterized in blood as $\mathrm{CD}^{+} \mathrm{c}^{+}$and $\mathrm{CD} 141^{+} \mathrm{mDCs}$ as well as $\mathrm{CD} 123^{+} \mathrm{pDCs}(9)$, infiltrate NPC lesions. $\mathrm{mDCs}$ and $\mathrm{pDCs}$, being antigen-presenting cells and representing an important association between innate and adaptive immunity, may thus be regarded as treatment targets in conditions such as NPC. Indeed, such a therapeutic potential is underscored by the present description of intralesional DC subsets, which may be selectively targeted to achieve a desired cross-presentation of antigens and resulting CTL responses. It may also be of interest to investigate the prognosis of NPC in association with the presence of DC subsets, due to conflicting information being presented for DCs (or DC-like cells) in this context $(18,22)$.

Considering DCs as treatment targets pertinent to NPC, it is important to reflect on how they can be instructed to facilitate cross-presentation of antigens. In this context, different DC subsets express distinct PRR profiles (9) and, in vaccination/active immunotherapy, adjuvant effects are mediated via these receptors. As a result, and depending on which PRR is activated, DCs receive directional information. A key consideration is that information on DC receptor repertories may be required to be subset specific. This is reflected by the fact that different DC populations may conduct opposing actions, including immunosuppression and immunoactivation. Accordingly, stimulation of a receptor that is not restricted to a specific DC subset may produce conflicting effects. Furthermore, experimental evidence indicates that stimulation of a number of receptors, including CD207, as demonstrated in CD1c ${ }^{+}$DCs in the present study, may facilitate cross-presentation of antigens (10).

The present study warrants future attempts to outline a complete map of PRRs on DC subpopulations in NPC and other head and neck cancer types, focusing on those PRRs that facilitate combined humoral and cellular antigen-specific responses, including TLR2, TLR4, Dectin-1, Dectin-2, CD206, DEC205, C-type lectin domain containing 9a, DC-specific intercellular adhesion molecule 3-grabbing non-integrin and $\mathrm{X}-\mathrm{C}$ motif chemokine receptor 1 (23). In the present study, the sample size was small, both in regard to patient numbers and tissue amount, reflecting the fact that this cancer type is rare and indicating that the number of cells available for flow cytometry analysis were too low for a wider profiling using this technique. Nevertheless, such examinations, and possibly a comparison to control tissue, are necessary and may, for example, be initiated by biomarker identification using single-cell RNA-sequencing methods (24). In this context, a comparison between $\mathrm{EBV}^{+}$and EBV- NPC may also be warranted as well as between NPC and control tissue.

In conclusion, a number of DC-subpopulations are present in NPC lesions. CLR CD207, as a selective endocytic marker on $\mathrm{CD}_{1} \mathrm{c}^{+} \mathrm{mDCs}$, may be targeted for therapeutic purposes to facilitate cross-presentation of antigens, serving potential cell-mediated antitumor effects. The present study leaves CD1c CD141 $\mathrm{mDCs}$, which have been recently identified $(24,25)$, open for further characterization, as they comprise a notable fraction of mDCs residing in the NPC samples.

\section{Acknowledgements}

The authors would like to thank Dr Fredrik Andersson (Department of Pathology, Skåne University Hospital, Lund, 
Sweden), for reviewing the histopathology results, and Dr Margareta Nilsson (Department of Diagnostic Radiology, Skåne University Hospital, Lund, Sweden), for reviewing the imaging results.

\section{Funding}

Funding was received from the Swedish National Health Service, the Swedish Association for Otorhinolaryngology Head and Neck Surgery, and Laryngfonden.

\section{Availability of data and materials}

The datasets used and/or analyzed during the current study are available from the corresponding author on reasonable request.

\section{Authors' contributions}

JSN, KL, ML and LG conceived the study. JSN conducted collection of samples and drafting of the manuscript. MA and KL conducted the laboratory work. All authors were involved in the preparation and revision of the manuscript.

\section{Ethics approval and consent to participate}

The study protocol was approved by the Ethics Committee at Lund University and written informed consent was obtained.

\section{Patient consent for publication}

Patient written consent for publication was obtained.

\section{Competing interests}

The authors declare that they have no competing interests.

\section{References}

1. Chua ML, Wee JT, Hui EP and Chan AT: Nasopharyngeal carcinoma. Lancet 387: 1012-1024, 2016.

2. Lee AW, Sze WM, Au JS, Leung SF, Leung TW, Chua DT, Zee BC, Law SC, Teo PM, Tung SY, et al: Treatment results for nasopharyngeal carcinoma in the modern era: The Hong Kong experience. Int J Radiat Oncol Biol Phys 61: 1107-1116, 2005.

3. Zackrisson B, Mercke C, Strander H, Wennerberg J and Cavallin-Ståhl E: A systematic overview of radiation therapy effects in head and neck cancer. Acta Oncol 42: 443-461, 2003.

4. Chua D, Huang J, Zheng B, Lau SY, Luk W, Kwong DL, Sham JS, Moss D, Yuen KY, Im SW and Ng MH: Adoptive transfer of autologous Epstein-Barr virus-specific cytotoxic T cells for nasopharyngeal carcinoma. Int J Cancer 94: 73-80, 2001.

5. Comoli P, Pedrazzoli P, Maccario R, Basso S, Carminati O, Labirio M, Schiavo R, Secondino S, Frasson C, Perotti C, et al: Cell therapy of stage IV nasopharyngeal carcinoma with autologous Epstein-Barr virus-targeted cytotoxic T lymphocytes. J Clin Oncol 23: 8942-8949, 2005.

6. Lin CL, Lo WF, Lee TH, Ren Y, Hwang SL, Cheng YF, Chen CL, Chang YS, Lee SP, Rickinson AB and Tam PK: Immunization with Epstein-Barr Virus (EBV) peptide-pulsed dendritic cells induces functional CD8 ${ }^{+} \mathrm{T}$-cell immunity and may lead to tumor regression in patients with EBV-positive nasopharyngeal carcinoma. Cancer Res 62: 6952-6958, 2002.
7. Smith C, Tsang J, Beagley L, Chua D, Lee V, Li V, Moss DJ, Coman W, Chan KH, Nicholls J, et al: Effective treatment of metastatic forms of Epstein-Barr virus-associated nasopharyngeal carcinoma with a novel adenovirus-based adoptive immunotherapy. Cancer Res 72: 1116-1125, 2012.

8. Collin M and Haniffa M: Dendritic cells and dendritic cell subsets. In: Encyclopedia of Immunobiology. Academic Press, Oxford, pp345-352, 2016.

9. Lundberg K, Rydnert F, Greiff L and Lindstedt M: Human blood dendritic cell subsets exhibit discriminative pattern recognition receptor profiles. Immunology 142: 279-288, 2014.

10. Joffre OP, Segura E, Savina A and Amigorena S: Cross-presentation by dendritic cells. Nat Rev Immunol 12: 557-569, 2012.

11. Valladeau J, Ravel O, Dezutter-Dambuyant C, Moore K, Kleijmeer M, Liu Y, Duvert-Frances V, Vincent C, Schmitt D, Davoust J, et al: Langerin, a novel C-type lectin specific to Langerhans cells, is an endocytic receptor that induces the formation of Birbeck granules. Immunity 12: 71-81, 2000.

12. Idoyaga J, Cheong C, Suda K, Suda N, Kim JY, Lee H, Park CG and Steinman RM: Cutting edge: Langerin/CD207 receptor on dendritic cells mediates efficient antigen presentation on MHC I and II products in vivo. J Immunol 180: 3647-3650, 2008.

13. Thomas JA, Iliescu V, Crawford DH, Ellouz R, Cammoun M and de-Thé G: Expression of HLA-DR antigens in nasopharyngeal carcinoma: An immunohistological analysis of the tumour cells and infiltrating lymphocytes. Int J Cancer 33: 813-819, 1984.

14. Braz-Silva PH, Vitale S, Butori C, Guevara N, Santini J, Magalhaes M, Hofman P and Doglio A: Specific infiltration of langerin-positive dendritic cells in EBV-infected tonsil, Hodgkin lymphoma and nasopharyngeal carcinoma. Int J Cancer 128: 2501-2508, 2011.

15. Hammar S, Bockus D, Remington F and Bartha M: The widespread distribution of Langerhans cells in pathologic tissues: An ultrastructural and immunohistochemical study. Hum Pathol 17: 894-905, 1986.

16. Lauriola L, Michetti F, Sentinelli S and Cocchia D: Detection of S-100 labelled cells in nasopharyngeal carcinoma. J Clin Pathol 37: 1235-1238, 1984.

17. Ma CX, Jia TC, Li XR, Zhand ZF and Yiao CB: Langerhans cells in nasopharyngeal carcinoma in relation to prognosis. In Vivo 9: 225-229, 1995.

18. Zong YS, Zhang CQ, Zhang F, Ruan JB, Chen MY, Feng KT and Yu ZF: Infiltrating lymphocytes and accessory cells in nasopharyngeal carcinoma. Jpn J Cancer Res 84: 900-905, 1993.

19. Thompson LD: Update on nasopharyngeal carcinoma. Head Neck Pathol 1: 81-86, 2007.

20. Sobin LH, Gospodarowicz MK and Wittekind C (eds): UICC: TNM Classification of Malignant Tumours. 7th edition. Wiley-Blackwell, New Jersey, 2009.

21. Segura E, Durand M and Amigorena S: Similar antigen cross-presentation capacity and phagocytic functions in all freshly isolated human lymphoid organ-resident dendritic cells. J Exp Med 210: 1035-1047, 2013.

22. Chang CS, Chang JH, Hsu NC, Lin HY and Chung CY: Expression of CD80 and CD86 costimulatory molecules are potential markers for better survival in nasopharyngeal carcinoma. BMC Cancer 7: 88, 2007.

23. Yin W, Duluc D, Joo H and Oh S: Dendritic cell targeting vaccine for HPV-associated cancer. Cancer Cell Microenviron 3: e1482, 2016.

24. Villani AC, Satija R, Reynolds G, Sarkizova S, Shekhar K, Fletcher J, Griesbeck M, Butler A, Zheng S, Lazo S, et al: Single-cell RNA-seq reveals new types of human blood dendritic cells, monocytes, and progenitors. Science 356: pii: eaah4573, 2017.

25. Abolhalaj M, Askmyr D, Sakellariou CA, Lundberg K, Greiff L and Lindstedt M: Profiling dendritic cell subsets in head and neck squamous cell tonsillar cancer and benign tonsils. Sci Rep 8: 8030, 2018. 